\title{
Specialized Pharmacy Oncology Technician: Experience at the Ridge Meadows Hospital
}

\author{
Anita Lo, Michelle Co, Chris Lo, Doson Chua, and David Soltesz
}

\section{INTRODUCTION}

$\mathrm{D}$ uring the past 40 years, the roles of the pharmacy technician have expanded to services beyond traditional duties within the pharmacy department, correlating with the ever-changing needs of the health care system. ${ }^{1}$ In other countries, such as the United Kingdom, the roles of pharmacy technicians go beyond those of their Canadian counterparts to include advising patients about medications, reviewing medication charts, and identifying medication-related problems, which can then be referred to pharmacists for resolution, which is more commonly known as follow-up for drug-related problems. ${ }^{2}$ In the United Kingdom, pharmacy technicians can also participate in discharge planning, can work in anticoagulation clinics, and can provide medication information to patients. ${ }^{3}$ In that country, voluntary registration to become a pharmacy technician started in 2005 and will be mandatory by 2011. ${ }^{4}$ Pharmacy technicians who are not registered by 2011 will no longer be able to call themselves pharmacy technicians or work in that capacity. In Canada, a national initiative is under way to regulate pharmacy technicians. Once regulated, pharmacy technicians will have an expanded scope of practice, for which they will be accountable and responsible. As in the United Kingdom, current technicians who choose not to go through the transition processes will not be able to call themselves pharmacy technicians. Starting with the definition of a national scope of practice in 2005, ${ }^{5}$ initiatives that have been explored include education outcomes, entry-to-practice competencies, accreditation of training programs, evaluation and entry-to-practice examinations, and bridging programs to support individuals currently employed as pharmacy technicians. The Canadian Council for the Accreditation of Pharmacy Programs is currently accrediting pharmacy technician programs in Canada in anticipation of the regulation of these allied health professionals.

In 2007, the Health Systems Improvement Act was passed by the Ontario Legislature, which enabled the regulation of pharmacy technicians by the Ontario College of Pharmacists. ${ }^{6}$ In British Columbia, initiatives are also under way for the regulation of technicians by the College of Pharmacists of British Columbia. The BC legislation allows pharmacists to manage, supervise, teach, or train others, including technicians, to provide pharmaceutical care. ${ }^{5}$

Recently, there was an opportunity to place a technician in the oncology unit at Ridge Meadows Hospital in Maple Ridge, British Columbia. No previous publications have described an enhanced role for technicians working in specialized areas. We describe here the unique position of a technician at Ridge Meadows Hospital who specializes as a clinical support technician in oncology and is known as the Specialized Pharmacy Oncology Technician or SPOT.

\section{DEVELOPMENT OF TECHNICIAN'S ROLE}

Ridge Meadows Hospital is a 302-bed community hospital. The outpatient oncology unit was created in 1997 with a focus on adult patients with solid tumours and malignant hematologic conditions. On average, 15 patients are booked to undergo chemotherapy in this outpatient unit each week. The oncology team, consisting of an oncologist, a nurse, a dietitian, a social worker, and a pharmacist, meets once a month to discuss patient care.

When the clinic was first created, the main roles of the pharmacist consisted of computer order entry, preparation of chemotherapy admixtures, and patient counselling. During the past few years, oncology protocols have become increasingly complex, and it became challenging to have one pharmacist perform all these tasks. Because recruitment of technicians is relatively easier than recruitment of pharmacists, ${ }^{7}$ it was suggested that a technician trained to a higher level of competency could help to ease the workload of the oncology pharmacist. The pharmacist could then focus on providing supportive care in the management of adverse effects of treatment, such as chemotherapyinduced nausea and vomiting, neutropenia, diarrhea, or anemia. 
A decision to create the SPOT position was made in October 2008, and the position was created shortly thereafter.

The candidate selected for the SPOT position (D.S.) was a graduate from a pharmacy technician program with 4 years of experience as a hospital pharmacy technician. The technician was also proficient in order entry and had been certified under the in-house tech-check-tech program. The SPOT had also completed a standard British Columbia Cancer Agency (BCCA) examination designed for pharmacists. In addition to preparing chemotherapeutic admixtures, a total of 6 functions were assigned to the SPOT: collecting laboratory data, printing patient profiles, performing calculations, triaging oncologyrelated calls, submitting drug approvals through the Compassionate Access Program, and interviewing patients.

\section{FUNCTIONS ASSIGNED TO SPOT}

\section{Collecting Laboratory Data}

The collection of laboratory data usually precedes dosage calculations and computer order entry, as laboratory values may dictate pertinent dosage changes or delays in therapy, according to treatment protocols. The SPOT was trained via a didactic lecture (given by the pharmacy manager/clinical practice leader, A.L.) based on the content of the pharmacy guide to chemotherapy protocols available at the BCCA website. ${ }^{8}$ The lecture emphasized the importance of understanding the relevance of counts of white blood cells, including neutrophils, red blood cells, and platelets; hemoglobin values; and other laboratory values reflecting renal and hepatic functions.

The SPOT is responsible for collecting the data through the hospital's laboratory system. The data are used routinely by the oncology pharmacist to assess the effectiveness of chemotherapy treatments.

\section{Printing Patient-Specific PharmaNet Profiles}

The SPOT is responsible for printing recent patient profiles from PharmaNet, the provincial drug database, which records medication orders filled outside the hospital. The oncology pharmacist reviews these profiles as part of monitoring each patient's medication regimen.

\section{Performing Calculations}

The SPOT calculates chemotherapeutic drug dosages before performing computer order entry. The pharmacist provides the training required to perform these complex calculations, which include determining body surface area and creatinine clearance. The SPOT is responsible for checking the dosage of each drug prescribed by the ordering oncologist on the basis of body surface area. One example of a complex calculation is the determination of carboplatin dosages using the
Calvert formula, where dose $(\mathrm{mg})=$ area under the curve $x$ (glomerular filtration rate +25 ). The SPOT is trained to double-check his own work. However, despite double-checking, errors have been made. Hence, as a final quality assurance step, the pharmacist verifies all calculations before order entry.

\section{Triaging Oncology-Related Calls}

All oncology-related calls are forwarded to the SPOT for triage according to the complexity of the questions. The SPOT answers simple questions relating to drug availability. $\mathrm{He}$ also gathers resources and looks into drug-drug interactions as requested by the physician, nurse, or patients. Finally, he reports his findings to the pharmacist before distributing the information.

\section{Making Requests to Compassionate Access Program and Performing Billings}

The Compassionate Access Program encompasses drugs for which special approval from the BCCA is required because the proposed regimen deviates from standard BCCA protocols.

The SPOT gathers all pertinent information, such as evidence of progression of disease and change of patient status from what is recorded in the patient's health record, to support the submission of such requests. He is trained to identify different types of protocols, to retrieve relevant information from patients' health records and submit it to the Compassionate Access Program within $24 \mathrm{~h}$ of the initial request, and to follow up on approval of the requests. In addition, the SPOT is assigned to perform monthly online billing and communicates with finance clerks if questions arise.

\section{Counselling Patients}

The SPOT participates in limited patient counselling, which is a vital component of successful chemotherapy. Reinforcing compliance with antiemetic therapy enhances the tolerability of chemotherapy for the patients. The oncology pharmacist creates a cue card with a list of questions for the SPOT to ask patients during these counselling sessions. Examples of questions are "Did you remember to take the pre-chemotherapy medications (for example, ondansetron or dexamethasone)?", "Do you have enough antiemetics or anti-diarrhea agents?", and "Do you have any problems with your medications?" Concerns requiring professional decisions are referred to the pharmacist. ${ }^{9}$ There are many other topics for which the SPOT can provide some patient counselling (e.g., management of anemia, management of pain, constipation, use of vitamins and herbal medications), but a discussion of these topics is beyond the scope of this article. 


\section{EVALUATION OF THE SPOT}

An evaluation of the SPOT is necessary to ensure patient safety and also to ensure that the duties performed by the technician are consistent with the standards that would be expected if the same duties were performed by a pharmacist. A formal evaluation process was still in development at the time this article was prepared, in late 2009. Ongoing quality assurance of the SPOT will be based on the Canadian Society of Hospital Pharmacists (CSHP) guidelines for delegation of functions to pharmacy technicians. ${ }^{10}$ However, some of the duties that the SPOT has undertaken go beyond those outlined in the current CSHP guidelines. The SPOT will be evaluated with respect to accuracy of recording laboratory data, ability to adhere to departmental policies and procedures when printing PharmaNet profiles, accuracy of pharmaceutical and chemotherapy calculations, appropriate referral of questions and concerns to a pharmacist, appropriate response to drug information requests, accuracy of documentation related to patient counselling, and ability to communicate with patients. It is intended that, initially, the manager should evaluate the progress and performance of the SPOT weekly, with input from the oncology nurse and physician, and identifies areas for improvement.

The weekly performance reviews conducted to date indicate that the SPOT has collected laboratory data accurately and has correctly followed policies and procedures for printing PharmaNet profiles. However, some mistakes have been made in pharmaceutical calculations, and the oncology working group has suggested that a pre-established formula be set up in the pharmacy computer for double-checking calculations. In addition, the group has suggested a review of the appropriateness of having the technician provide counselling to patients. For example, in interviewing patients, the SPOT should avoid asking questions about use of pre-chemotherapy medications if the chemotherapy infusion has already been started.

\section{DISCUSSION}

With the ongoing shortage of pharmacists, implementation of specialized roles for pharmacy technicians represents another way of freeing up pharmacists to perform the clinical duties for which they are trained. In addition to handling routine dispensary duties such as order entry and inventory management, 6 functions have been designated for the specialized technician at Ridge Meadows Hospital. Implementing the SPOT position has provided another independent check for calculations, which should reduce medication errors and improve patient safety. ${ }^{11}$ Quality assurance procedures, with the pharmacist performing a final check, are in place to ensure that the tasks delegated to the SPOT are performed as expected. Without such measures, the expanded roles of pharmacy technicians may pose risks to patients' safety.
Empowering technicians in specialized areas such as oncology requires that the technicians develop skills in therapeutics. In addition, support is needed to undertake further education and training. ${ }^{12}$ One institution has reported using a structured classroom program to train pharmacists and technicians. ${ }^{13}$ Our experience indicates that one-on-one training with a pharmacist and a technician is also feasible. The training has upgraded the technician's professional knowledge and has translated into great job satisfaction. The training has also enhanced communication among technicians, oncology nurses, and physicians, which has allowed integration of the technician into the collaborative environment of other health care professionals.

In the United States, technician certification programs are in place to ensure a recognized level of competence in the handling and preparation of medications. ${ }^{14,15}$ Certification programs in the area of oncology have recently been developed in British Columbia for consistent competency; these are focused mainly on preparing and handling antineoplastic drugs. ${ }^{16}$ Such certification programs in conjunction with the regulation of technicians in Canada will ensure the provision of safe, effective health care services, especially in specialty areas.

Given the impending regulation of technicians in some Canadian provinces, we hope to spark interest in supporting their future roles and to allow them to demonstrate their strengths in advancing their role as health care professionals.

\section{CONCLUSION}

The SPOT position has been a useful addition to the oncology service at Ridge Meadows Hospital.

\section{References}

1. Keresztes JM. Role of pharmacy technicians in the development of clinical pharmacy. Ann Pharmacother 2006;40(11):2015-2019.

2. Telford J, Soma N. Comparing the role of pharmacy technicians in the US and UK. Hosp Pharm 2005;12(9):379-380.

3. Thomas M, Cross S, Nagra M, Bullock A. Empowering technicians to work in extended clinical roles. Hosp Pharm 2008;15(4):145-146.

4. Flint J. Regulation of pharmacy technicians. London (UK): Royal Pharmaceutical Society of Great Britain; [cited 2009 Mar 19]. Available from: www.rpsgb.org/pdfs/educpolfitforfutregphtech.pdf

5. Pharmacy technician regulation. Vancouver (BC): College of Pharmacists of British Columbia; [cited 2009 Mar 19]. Available from: www.bcpharmacists. org/about_us/key_initiatives/index/articles27.php\#Background

6. Regulation of pharmacy technicians. Toronto (ON): Ontario College of Pharmacists; 2009 [cited 2009 Mar 19]. Available from: http:// www.ocpinfo.com/client/ocp/OCPHome.nsf/web/Regulation+of+ Pharmacy+Technicians

7. Babich M. Human resources. In: Babich M, Bussières JF, Hall KW, Harding J, Johnson N, Lefebvre P, et al., editors. Hospital pharmacy in Canada: 2007/2008 report. Eli Lilly; 2008 [cited 2009 Mar 19]. p. 42-52. Available from: www.lillyhospitalsurvey.ca/hpc2/content/2008_report/ humanresources.pdf

8. Annable D, Cassidy S, Coady N, Stronach A. Chemotherapy protocols: the clinical interpretation and application of treatment protocol summaries. Vancouver (BC); BC Cancer Agency; 2003 [republished 
online 2010; cited 2009 Mar 19]. Available from: www.bccancer.bc.ca/ NR/rdonlyres/A36F6632-B25A-4321-8920-60687EEF20CF/43657/ ProtocolGuide06Jan10.pdf

9. Section 83. Pharmacy support assistant. In: Bylaw of the Council of the College of Pharmacists of British Columbia. Vancouver (BC): College of Pharmacists of British Columbia; 1999 [cited 2009 Mar 24]. Available from: www.bcpharmacists.org/legislation/provincial/bylaws

10. Pharmacy technicians: guidelines on the delegation of functions to pharmacy technicians. Ottawa (ON): Canadian Society of Hospital Pharmacists; 2006

11. Cohen MR, Anderson RW, Attilio RM, Green L, Muller RJ, Pruemer JM. Preventing medication errors in cancer chemotherapy. Am J Health Syst Pharm 1996;53(7):737-746.

12. Moving forward: pharmacy human resources for the future. Ottawa (ON): Canadian Pharmacists Association; 2008 [cited 4 Apr 2009]. Available from: www.pharmacyhr.ca

13. Peters BG, Wilson AL, Lunik MC, Cataldo BK. Certification program in antineoplastic drug preparation for pharmacy technicians and pharmacists. Am J Hosp Pharm 1994;51(15):1902-1906.

14. Gans JA, Manasse HR Jr. Certification of pharmacy technicians. Am J Health Syst Pharm 2008;65(19):1798-1800.

15. Muenzen PM, Corrigan MM, Smith MA, Rodrigue PG. Updating the pharmacy technician certification examination: a practice analysis study. Am J Health Syst Pharm 2005;62(23):2542-2546.
16. Waddell JA, Hannan LC, Stephens MR. Pharmacy technician competencies for practice in an oncology pharmacy. J Pharm Technol 1998;14:191-201.

Anita Lo, BSc(Pharm), PharmD, is Pharmacy Manager/Clinical Practice Leader, Eagle Ridge Hospital, Port Moody, British Columbia.

Michelle Co, BSc(Pharm), is a Pharmacist, Ridge Meadows Hospital, Maple Ridge, British Columbia.

Chris Lo, BSc(Pharm), PharmD, is Clinical Practice Leader, Langley Memorial Hospital, Langley, British Columbia.

Doson Chua, PharmD, BCPS(AQ), is a Clinical Pharmacy Specialist, St Paul's Hospital, Vancouver, British Columbia.

David Soltesz is a Pharmacy Technician, Ridge Meadows Hospital, Maple Ridge, British Columbia.

Address correspondence to:

DrAnita Lo

Eagle Ridge Hospital

475 Guildford Way

Port Moody BC V3H 3W9

e-mail: anita.lo@fraserhealth.ca 